\title{
The relation of autologous serum and plasma skin test results with urticarial activity score, sex and age in patients with chronic urticaria
}

\author{
Sirac Aktar ${ }^{1}$, Necmettin Akdeniz², Hatice Uce Ozkol ${ }^{3}$, Omer Calka ${ }^{3}$, Ayse Serap Karadag² \\ ${ }^{1}$ Department of Dermatology, Diyarbakır Research and Training Hospital, Diyarbakir, Turkey \\ Head of the Department: Sirac Aktar \\ 2Department of Dermatology, Faculty of Medicine, Medeniyet University, Goztepe Training and Research Hospital, Istanbul, Turkey \\ Head of the Department: Necmettin Akdeniz \\ ${ }^{3}$ Department of Dermatology, Faculty of Medicine, Yuzuncu Yil University, Van, Turkey
}

Head of the Department: Omer Calka

Postep Derm Alergol 2015; XXXII (3): 173-178

DOI: 10.5114/pdia.2015.48057

\begin{abstract}
Introduction: Some previous studies reported autoimmunity as an etiologic factor in chronic urticaria (CU), but the results of some autoimmunity tests in these studies are conflicting.

Aim: To concretize whether there was any relation of autologous serum skin test (ASST) and autologous plasma skin test (APST) results with sex, age and urticarial activity score (UAS) in patients with CU.

Material and methods: Fifty patients with CU and twenty healthy subjects admitted to our dermatology clinic were included in the present study. The ASST and APST were applied to all individuals.

Results: The positiveness rates of ASST and APST were significantly higher in the patient group than controls ( $p=0.027, p=0.001$, respectively). Among patients, the APST positiveness rate $(72 \%)$ was significantly $(p<0.05)$ higher than ASST (46\%). It was seen that $48 \%$ of patients with negative ASST results had positive APST. However, no patient with negative APST results had positive ASST. There were significant $(p<0.05)$ relations of the tests' positiveness rates with sex and old age but with UAS. The diameter of the erythematous papule was remarkably $(p<0.05)$ larger in APST than ASST and also significantly $(p<0.05)$ larger in females compared to males in both tests $(p<0.05)$. It was positively increased with old age $(p<0.05)$.

Conclusions: We can suggest that APST is more sensitive than ASST in the assessment of autoimmunity in CU. A high positiveness rate of APST results may be attributed to high numbers of autoantibodies and coagulation factors present in plasma that might probably play a role in etiopathogenesis of CU.
\end{abstract}

Key words: chronic urticaria, autoimmunity, autologous serum skin test, autologous plasma skin test.

\section{Introduction}

Urticaria is a frequently seen vascular reaction of skin and mucosa. It is characterized by transient erythematous and edematous as well as raised skin plaques. Acute urticaria is usually seen in young patients, whereas chronic urticaria (CU) is commonly seen in middle-aged women. If urticaria lasts more than 6 weeks, it is classified as CU [1]. Chronic urticaria is divided into two groups: 1) chronic autoimmune urticaria (CAU) (45\%); and 2) chronic idiopathic urticaria (CIU) (55\%) [2].
In recent years, autoimmunity has been reported as an etiologic factor in $40-60 \%$ of CIU cases [3]. IgG type autoantibodies that are developed against an $\alpha$ subunit of the high affinity IgE receptors in mast cells and basophiles were detected in 15-31\% of the cases [4]. The positiveness rate of this autoantibody was detected in around 37-60\% among CU cases in different studies [5, 6]. It was shown that autoantibodies that are developed against FceRl $\alpha$ and IgE were IgG1 and IgG3 isotypes [7]. These aforementioned cases were named as CAU.

Address for correspondence: Hatice Uce Ozkol, Department of Dermatology, Faculty of Medicine, Yuzuncu Yil University, Zeve Campus, 65087 Van, Turkey, phone: +90 5058313536, e-mail: drhaticeuce@gmail.com Received: 6.08.2014, accepted: 24.09.2014. 
The autoantibodies were shown to trigger histamine release of basophiles (from a healthy donor) and mast cells in vitro $[4,8]$. The gold standard test for the functional autoantibodies in CAU is a basophil histamine release test. However, due to difficulties in standardization and a prolonged duration of the test, it is usually used for advanced researches. Another diagnostic test for CAU is an autologous serum skin test (ASST). Application of this test is simple, easier, cheaper, and more accurate than the basophil histamine release test. An in vivo test ASST is analogous of in vitro basophil histamine release test and it is based on formation of erythema and swelling after intradermal autologous serum injection [7]. This test was the first observation to help us define antibodies in circulating blood [9]. The simple observation shows that histamine releasing factors are present in the patients' serum that may be related to the presence of functional anti-FceRl $\alpha$ and anti IgE antibodies in CAU $[4,5,10]$. The ASST is a simple method for the diagnosis of autoreactive CU, but it can cause false positive results, which is related to the formation of a large amount of bradykinin and secretion of tryptases from neutrophils during the coagulation process [11, 12]. In a double blind placebo controlled study it was shown that warfarin (an anticoagulation medication) provided a remarkable response in anti-histamine resistant CU cases [13].

In a previous study, presence of antibodies, coagulation factors and some other proteins were reported to be higher in the plasma than serum $[14,15]$. Plasma thrombin production increases endothelial cell permeability and may lead to activation of mast cells and extrinsic coagulation pathway in CU. Increased tissue factors were shown in skin biopsies of CU patients [11]. Some factors occurring in the course of coagulation may cause false positive results of ASST, thus the specificity of ASST has been controversial in the diagnosis of CAU $[7,16]$. Therefore, it has been recently suggested that APST may be more sensitive than ASST [17]. Heparin can inhibit in vivo and in vitro degranulation of mast cells and basophiles, so if plasma is anti-coagulated with heparin, it cannot be used for APST $[18,19]$. Thus plasma that was anti-coagulated with sodium citrate was used for APST.

\section{Aim}

In this study, we aimed to concretize whether there was a relation of APST and ASST results of CU patients with age, sex and urticarial activity score (UAS) because the results of a few previous studies in this regard were conflicting.

\section{Material and methods}

Fifty patients who were admitted to an dermatology outpatient clinic between April 2009 and January 2010 and had chronic or recurrent urticaria with or without angioedema for at least 6 weeks were enrolled in our study (18 male and 32 female; mean \pm SD age $29.72 \pm 8.52$ years, range: $16-51)$. Twenty healthy individuals were selected as control subjects ( 8 males and 12 females; mean \pm SD age $26.40 \pm 8.27$ years, range: $15-43$ ). These control subjects had no history of urticaria, allergic and autoimmune diseases and psychiatric treatment. In addition, patients who had physical urticaria and urticaria continued for less than 6 weeks as well as who used urticaria induced drugs or immunosuppressive drugs within last 3 months were excluded from the study. We defined UAS of the subjects. An informed consent form was obtained from all subjects, and the study was conducted based on the Good Clinical Practice guidelines. The study was approved by the local ethics committee and conducted according to the ethical principles of the Declaration of Helsinki.

\section{Application of Autologous Serum and Plasma Skin Tests}

Tests (ASST and APST) were applied on the flexor surfaces of both forearms of patients with CU and the controls. Antihistaminic drugs were stopped at least 3 days and mast cell stabilizers were stopped at least 7 days before the tests. Totally, a $5 \mathrm{ml}$ sample of venous blood was collected from each subject. Two $\mathrm{ml}$ of blood were taken in a tube with sodium citrate and the rest in a biochemistry tube. After $30 \mathrm{~min}$ in room temperature, the samples were centrifuged (Jouanbrand CR4i, France) with 4000 rpm for $7 \mathrm{~min}$ and then serum and plasma were taken.

Autologous plasma, undiluted autologous serum, and $0.9 \%$ saline were taken with separate insulin syringes (0.1 cc from each solution) and were applied on the flexor surfaces of both forearms. The injections were applied on the areas that did not have any urticarial papules within last $24 \mathrm{~h}$. There was at least $5 \mathrm{~cm}$ distance between the injection areas. The results were assessed 30 min after the application. When the diameter of the erythematous papule formed by autologous serum or plasma was at least $1.5 \mathrm{~mm}$ larger than the control area where 0.9\% saline was administered, the response was accepted as positive. The diameter was measured by a digital caliper. Before applying the tests, adrenaline, injectable prednisolone, and antihistamines were prepared ready for a possible anaphylactic reaction. The tests were applied by one researcher and the results were interpreted by another researcher.

\section{Assessment of Urticarial Activity Score}

The severity of disease was assessed by UAS as recommended by the EAACI/GA2LEN/EDF guidelines [20]. UAS was calculated as the sum of the score values for 
the number of wheals and pruritus intensity. The total score ranges from 0 to 6.

\section{Statistical analysis}

Descriptive statistics for studied variables (characteristics) were presented as mean, standard deviation, minimum and maximum values. In addition, categorical variables were described with the number and percentage. Student $t$ test was used to compare control and patient groups' means for the studied continuous variables. For determination of linear relationships among these variables, Pearson correlation analysis was carried out. Furthermore, the $\chi^{2}$ test was utilized for determination of the relationships between categorical variables. Statistical significance levels were considered as 5\% and SPSS (ver. 13) statistical program was used for all statistical computations.

\section{Results}

Sex, age and test results of the patient and control groups are summarized in Table 1. The positiveness rates of ASST and APST were significantly higher in the patient group than controls ( $p=0.027, p=0.001$, respectively). As shown in Table 2, the positiveness rates of ASST and APST increased with old age ( $p=0.028, p=0.027$, respectively) and were remarkably higher in female subjects ( $p=0.001$ for ASST, $p=0.032$ for APST). Twenty three patients (46\%) had positive ASST and 36 (72\%) had positive APST. The positiveness rate of APST was determined to be significantly $(p<0.05)$ higher than ASST
Table 1. Comparison of the features and the results of tests between the $\mathrm{CU}$ and the control groups

\begin{tabular}{lccc}
\hline Feature & $\begin{array}{c}\text { The patient } \\
\text { group }\end{array}$ & $\begin{array}{c}\text { Control } \\
\text { group }\end{array}$ & Value of $p$ \\
\hline Number of patients & 50 & 20 & \\
\hline $\begin{array}{l}\text { Mean age, mean } \pm \\
\text { standard deviation }\end{array}$ & $29.72 \pm 8.52$ & $26.40 \pm 8.27$ & 0.823 \\
\hline $\begin{array}{l}\text { Gender: } \\
\text { Male }\end{array}$ & $18(36 \%)$ & $8(40 \%)$ & 0.754 \\
\hline Female & $32(64 \%)$ & $12(60 \%)$ & \\
\hline APST positive & $36(72 \%)$ & $3(15 \%)$ & 0.001 \\
\hline APST negative & $14(28 \%)$ & $17(85 \%)$ & 0.001 \\
\hline ASST positive & $23(46 \%)$ & $3(15 \%)$ & 0.027 \\
\hline ASST negative & $27(54 \%)$ & $17(85 \%)$ & 0.027 \\
\hline ASST - autologous serum skin test, APST-autologous plasma skin test.
\end{tabular}

(Table 3). When patients who had negative ASST results were examined, it was seen that $48 \%$ of them had positive APST results. However, all the patients with negative APST results had also negative ASST results. As seen in Table 4, the diameter of the erythematous papule was remarkably larger in APST than ASST and also significantly larger in females compared to males in both tests $(p<0.05)$. In addition, both diameters of ASST and APST had a significant positive relation with old age $(p<0.05)$ (Table 5). As shown in Table 6, no significant relation was determined between UAS and results of both tests ( $p=0.909$ for ASST, $p=0.262$ for APST). Furthermore,

Table 2. Comparison of sex and age between ASST and APST positive and negative groups

\begin{tabular}{lccccc}
\hline \multirow{2}{*}{ Variable } & \multicolumn{2}{c}{ ASST } & Value of $p$ & \multicolumn{2}{c}{ APST } \\
\cline { 2 - 5 } & Positive & Negative & & Positive & Negative \\
\hline Number of patients & 23 & 27 & 36 & 14 \\
\hline $\begin{array}{l}\text { Age, } \\
\text { mean } \pm \text { standard deviation }\end{array}$ & $35.65 \pm 9.12$ & $29.59 \pm 9.66$ & 0.028 & $34.28 \pm 9.29$ & $27.50 \pm 9.73$ \\
\hline Male/female & $5 / 18$ & $13 / 14$ & 0.001 & $9 / 27$ & $9 / 5$ \\
\hline
\end{tabular}

Table 3. Comparison of the results of ASST and APST

\begin{tabular}{lccc} 
& $\begin{array}{c}\text { APST } \\
\text { negative }\end{array}$ & $\begin{array}{c}\text { APST } \\
\text { positive }\end{array}$ & Total \\
\hline ASST negative & $14(52 \%)$ & $13(48 \%)$ & $27(54 \%)$ \\
\hline ASST positive & 0 & $23(100 \%)$ & $23(46 \%)$ \\
\hline Total & $14(28 \%)$ & $36(72 \%)$ & $50(100 \%)$ \\
\hline
\end{tabular}

Table 4. Comparison of the diameter of ASST and APST according to sex from both forearms in the CU group

\begin{tabular}{llcc}
\hline Variable & & Mean \pm SD $[\mathrm{mm}]$ & Value of $p$ \\
\hline \multirow{2}{*}{ Left ASST } & Male & $1.85 \pm 2.83$ & 0.045 \\
\cline { 2 - 3 } & Female & $3.83 \pm 3.49$ & \\
\hline \multirow{2}{*}{ Reft APST } & Male & $3.13 \pm 3.21$ & 0.003 \\
\cline { 2 - 3 } & Female & $6.36 \pm 3.55$ & \\
\hline Right ASST & Male & $1.83 \pm 2.76$ & 0.039 \\
\cline { 2 - 3 } & Female & $3.89 \pm 3.54$ & \\
\cline { 2 - 3 } & Male & $3.03 \pm 3.27$ & 0.002 \\
\hline
\end{tabular}


Table 5. The correlation between the diameter of the ASST and APST response, and age from both forearms in the CU group

\begin{tabular}{lc}
\hline Diameter of the test & Age \\
\hline Left ASST & $0.327^{\star}$ \\
\hline Left APST & $0.343^{*}$ \\
\hline Left SP & 0.169 \\
\hline Right ASST & $0.324^{\star}$ \\
\hline Right APST & $0.367^{\star \star}$ \\
\hline Right SP & 0.157 \\
\hline
\end{tabular}

$S P-$ Serum physiologic. ${ }^{*} p<0.05,{ }^{* *} p<0.01$.

disease duration was also found unrelated to ASST and APST results.

\section{Discussion}

In this study, we investigated possible relations of ASST and APST results with sex, age and UAS in patients with CU. In some previous studies, urticaria was informed to be seen two times more frequently in women than men [21]. In accordance with these data, $64 \%$ of the subjects were women and $36 \%$ of them were men in the patient group of our study. Chronic urticaria is frequently seen in middle-aged patients, particularly in the $3^{\text {rd }}$ and $4^{\text {th }}$ decades $[22,23]$. The mean age of patients with CU was $29.72 \pm 8.525$ years in our study. The mean duration of CU was around 3 years in the present study while it was reported to be 4 years in Metz et al.'s study [22]. We did not find any relations of age or disease duration with both sexes.

The ASST shows presence of functional autoantibodies in CU [4, 24]. There is a discrepancy in its reported results with some earlier studies. Grattan et al. [25] in which ASST was applied for the first time to patients with CU reported $58 \%$ of positive results. The positiveness rate of ASST was found to be $60 \%$ in a study of Niimi et al. [26] while $17 \%$ in Harmanyeri et al. [24] and $40 \%$ in $\mathrm{Hi}$ zal et al. [27]. With regard to our study, this rate of ASST was $46 \%$. We measured the diameter of erythematous papules with a digital caliper in accordance with studies of Sabroe et al. [4] and Harmanyeri et al. [24]. The results were accepted as positive when the erythematous diameter was at least $1.5 \mathrm{~mm}$ larger in ASST compared to the control area where $0.9 \%$ saline was administered.

In the present study, we included 20 healthy subjects in the control group. Positiveness rates of APST and ASST were the same (15\%) in this group. Apart from our study, Alpay et al. [28] and Yildiz et al. [14] found positiveness rates in the control group as $10 \%$ and $30 \%$, respectively. In our study, the diameter of erythematous papules of ASST and APST were remarkably higher in female patients than males and there was a positive relation of this
Table 6. Comparison of UAS with ASST and APST results

\begin{tabular}{lcc}
\hline & $\begin{array}{c}\text { UAS, mean } \pm \mathrm{SD}, \\
\text { range } 0-6\end{array}$ & Value of $p$ \\
\cline { 1 - 2 } ASST-negative & $3.15 \pm 1.74$ & 0.909 \\
\hline ASST-positive & $3.09 \pm 2.02$ & \\
\hline APST-negative & $2.64 \pm 1.39$ & 0.262 \\
\hline APST-positive & $3.31 \pm 1.99$ & \\
\hline
\end{tabular}

UAS - urticarial activity score.

diameter with old age. In contrast to our study, Aytekin et al. [29] suggested a significant increment of ASST positivity with young age and no remarkable relationship was reported between this positivity and sex in that study. Kocatürk et al. [30] showed no remarkable difference of ASST and APST test results with age, sex, disease duration, UAS and presence of angioedema or other autoimmune diseases in patients with CU. We applied foregoing tests on both forearms of subjects for the first time in the literature. However, we could not find any remarkable difference between the right and left forearms.

In the patient group of our study, ASST and APST positiveness rates were $46 \%$ and $72 \%$, respectively. When patients who had negative ASST results were evaluated, it was seen that $48 \%$ of them had positive APST results. However, all the patients with negative APST results had also negative ASST results. In a study of Asero et al. [11] which was conducted on 96 patients with CU, ASST and APST tests were compared. The positiveness rate of ASST was $53 \%$ and APST was $86 \%$. In another recent study conducted on 200 patients with CU, ASST and APST positiveness rates were $37.5 \%$ and $43 \%$, respectively and $23.2 \%$ of patients with negative ASST results had positive APST [22]. Unlike the results of our study, Kocatürk et al. [30] reported a higher ASST positiveness rate (62.9\%) than APST (38.6\%). However, Alpay et al. [28] and Yildiz et al. [14] reported approximately equal results of APST and ASST positiveness rates. Our current results are consistent with Asero et al. [11] and Metz et al. [22] in which higher numbers of patients were evaluated. These results suggest that APST is more sensitive than ASST.

According to the results of the current study, the diameter of the erythematous papule was remarkably larger in APST than ASST and also significantly larger in females compared to males in both tests. Our bigger result of the diameter in APST comparing to ASST was also reported in Godse [23]. However, Asero et al. [11] reported a slightly larger diameter of APST than ASST. A larger diameter of APST detected in our study may have resulted from coagulation factors or high rate autoantibodies in plasma.

Generally, the severity of urticaria is assessed by UAS as recommended by the EAACI/GA2LEN/EDF guidelines [20]. Urticarial activity score was reported to be signifi- 
cantly higher in patients with positive ASST results than patients with negative ASST in a study of Metz et al. [22]. However, there was no statistically significant difference in UAS between patients with APST positive and negative results in that study. With regard to our current trial, there was no remarkable difference of UAS between the patients with positive and negative ASST results in line with Kocatürk et al. [30]. We found similar results between UAS and APST.

In the present trial, duration of CU was detected to be unrelated to ASST and APST results. In line with our study, Hizal et al. [27] and Kocatürk et al. [30] did not find any significant differences in the duration of disease between ASST positive and negative groups. Unlike these foregoing studies, Grattan et al. [25] showed that patients with positive ASST results had shorter duration of disease than the patients with negative ASST results.

In the recent years, it has been asserted that thrombocyte-derived factors and other coagulation factors may play an important role in skin reactions and pathogenesis of CU [11]. Complement cascades were observed to have an important role in mast cells degranulation [31]. Asero et al. [32] showed that the levels of D-dimer, $\mathrm{F}_{1+2}$, and FVII were significantly higher in the CU patients with active lesions than the healthy subjects. Yıldız et al. [14] also reported similar results. These findings suggested that the external coagulation system might be active in patients with CU. The last product of intrinsic and extrinsic coagulation systems is thrombin. Thrombin may induce C5a (anaphylatoxin) production, permeability of vessels, mast cells activation and degranulation $[19,33]$. Thus, it has been suggested that thrombin might play an active role in the pathogenesis of CU [34]. Acute angioedema attacks were associated with the excessive thrombin production either in hereditary or acquired $\mathrm{C} 1$ inhibitor deficiency [11]. Coagulation factors, autoantibodies, and histamine releasing factors are present in plasma. However, no coagulation factors are present in serum. Therefore, coagulation factors induced skin reaction cannot be seen in ASST and this case may cause false negative results [14]. Due to the presence of coagulation factors in plasma, APST provides more information on the pathogenesis of urticaria. Moreover, an earlier study which found warfarin to be effective in treatment of CU (antihistaminic drug resistant) supported foregoing roles of coagulation factors in CU [13].

\section{Conclusions}

The APST positiveness results of patients with CU were remarkably higher than ASST. In addition, $48 \%$ of the patients with negative ASST results had positive APST indicating the roles of coagulation factors and high antibody content of plasma in the etiopathogenesis of CU. Moreover, we found a remarkably larger diameter of the erythematous papule in APST than ASST in both sexes. High positiveness rates of tests and the larger diameter in females may be attributed to high rates of autoimmune diseases seen in women. Therefore, in order to evaluate autoimmunity of CU, APST can be more sensitive than ASST. Further studies investigating anticoagulant medications in the treatment of $\mathrm{CU}$ are needed to enlighten our current findings.

\section{Acknowledgments}

We would like to thank Associate Professor Halil Ozkol for reviewing the manuscript.

\section{Conflict of interest}

The authors declare no conflict of interest.

\section{References}

1. Asero R, Tedeschi A, Cugno M. Treatment of refractory chronic urticaria: current and future therapeutic options. Am J Clin Dermatol 2013; 14: 481-8.

2. Kaplan AP. Urticaria and angioedema. In: Fitzpatrick's dermatology in general medicine. Wolff K, Goldsmith LA (eds). 7th ed. Mcgraw-Hill, New York 2008; 330-43.

3. Najib U, Sheikh J. The spectrum of chronic urticaria. Allergy Astma Proc 2009; 30: 1-10.

4. Sabroe RA, Grattan CE, Francis DM, et al. The autologous serum skin test: a screening test for autoantibodies in chronic idiopathic urticaria. Br J Dermatol 1999; 140: 446-52.

5. Leznoff A, Sussman GL. Sydrome of idiopathic chronic urticaria and angioedema with thyroid autoimmunity: a study of 90 patients. J Allergy Clin Immunol 1989; 84: 66-71.

6. Fiebiger E, Maurer D, Holub H, et al. Serum IgG autoantibodies directed against the a chain of FCepsilonRI: a selective marker and pathogenetic factor for a distinct subset of chronic urticaria patients. J Clin Invest 1995; 96: 2606-12.

7. Grattan CH, Sabroe RA, Greaves MW. Chronic urticaria. J Am Acad Dermatol 2002; 46: 645-57.

8. Asero R, Lorini M, Chong SU, et al. Assessment of histamine-releasing activity of sera from patients with chronic urticaria showing positive autologous skin test on human basophils and mast cells. Clin Exp Allergy 2004; 34: 1111-4.

9. Sabroe RA, Greaves MW. Chronic idiopathic urticaria with functional autoantibodies: 12 years on. Br J Dermatol 2006; 154: 813-9.

10. Hide M, Francis DM, Grattan CEH, et al. Autoantibodies against the high affinity IgE reseptor as a cause of histamine release in chronic urticaria. N Engl J Med 1993; 328: 1599-604.

11. Asero R, Tedeschi A, Riboldi P, Cugno M. Plasma of patients with chronic urticaria shows signs of thrombin generation, and its intradermal injection causes wheal-and-flare reactions much more frequently than autologous serum. J Allergy Clin Immunol 2006; 117: 1113-7.

12. Kaplan AP, Joseph K, Shibayama Y, et al. Bradykinin formation: plasma and tissue pathways and cellular interactions. In: Inflammation: basic principles and clinical correlates. Gallin J, Snyderman R (eds). 3rd ed. Lippincott, Williams and Wilkins, Philadelphia 1999; 331-47.

13. Parslew R, Pryce D, Ashworth J, Friedmann PS. Warfarin treatment of chronic idiopathic urticaria and angio-oedema. Clin Exp Allergy 2000; 30: 1161-5. 
14. Yıldız H, Karabudak O, Doğan B, Harmanyeri Y. Evaluation of autologous plasma skin test in patients with chronic idiopathic urticaria. Br J Dermatol 2011; 165: 1205-9.

15. Scott Y, Parker P, McArdle B, Wallis JP. Comparison of plasma and serum for antibody detection using DiaMedmicrotubes. Transfus Med 1996; 6: 65-7.

16. Grattan CEH. Autoimmune urticaria. Immunol Allergy Clin N Am 2004; 24: 163-81.

17. Asero R, Cugno M, Tedeschi A. Autologous plasma and serum skin test in chronic urticaria. $\mathrm{Br}$ J Dermatol 2012; 166: 1362-3.

18. Asero R, Cugno M, Tedeschi A. Activation of blood coagulation in plasma from chronic urticaria patients with negative autologous plasma skin test. J Eur Acad Dermatol Venereol 2011; 25: 201-5.

19. Asero R, Tedeschi A, Lorini M, et al. Chronic urticaria: novel clinical and serological aspects. Clin Exp Allergy 2001; 31: 1105-10.

20. Zuberbier T, Bindslev-Jensen C, Canonica W, et al. EAACI/GA2LEN/EDF. EAACI/GA2LEN/EDF guideline: definiation, classification and diagnosis of urticaria. Allergy 2006; 61: 316-20.

21. Sibbald RG, Cheema AS, Lozinski A, Tarlo S. Chronic urticaria: evaluation of the role of physical, immunologic and other contributory factors. Int I Dermatol 1991; 30: 381-6.

22. Metz M, Gimenez-Arnau A, Borzova E, et al. Frequency and clinical implications of skin autoreactivity to serum versus plasma in patients with chronic urticaria. J Allergy Clin Immunol 2009; 123: 705-6.

23. Godse KV. Autologous serum skin test v/s autologous plasma skin test. Indian J Dermatol Venerol Leprol 2008; 74: 496-7.

24. Harmanyeri Y, Dogan B, Taskapan MO, Oz M. Autologous serum skin test: prevalence study in patients with chronic idiopathic urticaria. Turkderm 2000; 34: 93-4.

25. Grattan CEH, Wallington TB, Warin RP, Kennedy CTC. A serological mediator in chronic idiopathic urticaria: a clinical, immunological and histological evaluation. $\mathrm{Br} J$ Dermato 1986; 114: 583-90.

26. Niimi N, Francis DM, Kermani F, et al. Dermal mast cell activation by autoantibodies against the high affinity lgE receptor in chronic urticaria. J Invest Dermatol 1996; 106: 1001-6.

27. Hizal M, Tüzün B, Wolf R, Tüzün Y. The relationship between Helicobacter pylori IgG antibody and autologous serum test in chronic urticaria. Int I Dermatol 2000; 39: 443-5.

28. Alpay A, Solak Tekin N, Tekin IÖ, et al. Autologous serum skin test versus autologous plasma skin test in patients with chronic spontaneous urticaria. Dermatol Res Pract 2013; 2013: 267278.

29. Aytekin S, Turkmen H. Investigation of autologous serum skin test and etiological factors in 31 patients with chronic urticaria. Turkiye Klinikleri Dermatol 2001; 11: 141-5.

30. Kocatürk E, Kavala M, Kural E, et al. Autologous serum skin test vs autologous plasma skin test in patients with chronic urticaria: evaluation of reproducibility, sensitivity and specificity and relationship with disease activity, quality of life and anti-thyroidantibodies. Eur J Dermatol 2011; 21: 339-43.

31. Fusari A, Colangelo C, Bonifazi F, Antonicelli L. The autologous serum skin test in the follow-up of patients with chronic urticaria. Allergy 2005; 60: 256-8.

32. Asero R, Tedeschi A, Coppola R, et al. Activation of the tissue factor pathway of blood coagulation in patients with chronic urticaria. J Allergy Clin Immunol 2007; 119: 705-10.
33. Cirino G, Cicala C, Bucci MR, et al. Trombin functions as an inflammatory mediator through activation of its receptor. J Exp Med 1996; 183: 821-7.

34. Asero R, Riboldi P, Tedeschi A, et al. Chronic urticaria: a disease at a crossroad between autoimmunity and coagulation. Autoimmun Rev 2007; 7: 71-6. 